\title{
Nervous Control of the Renal Tubular Function
}

\author{
Jugoro Takeuchi, M.D., Nobuo Ohya, M.D., Shuichiro Sakai, M.D., \\ Haruo Nakamura, M.D., Tetsuo Nohara, M.D., \\ Kunihiko Hirasawa, M.D., and Akira Shinoda, M.D.
}

\section{SUMmaRY}

The role of the renal nerves in renal tubular function-which is still a controversial subject-was studied by observing the changes in renal nerve activity and urine formation before, during and after the left stellate ganglion stimulation. The experiments were performed on 7 dogs anesthetized with a combination of morphine and $\alpha$-chloralose. The activity of one of the renal nerves was picked up by a platinum bipolar electrode and recorded. During the left stellate ganglion stimulation, the rate of discharge of renal nerve activity decreased in the number of bursts and, at the same time, increascs in urine volume and excretion of sodium, potassium and solutes were observed, while the urinary concentrations of sodium, potassium and osmolality decreased. These alterations produced by stellate ganglion stimulation were characterized by a rapid onset and, with cessation of stimulation, a rapid disappearance. There was observed complete recovery after the stimulation. On the other hand, the estimation of glomerular filtration rate revealed no significant changes through each experimental period.

The mechanisms of stellate ganglion stimulation diuresis were discussed and it was assumed that there was nervous control operating on renal tubular function, especially in the proximal tubules.

\section{Additional Indexing Words :}

Renal nerve activity Stellate ganglion stimulation diuresis Bipolar electrode Sodium transport Sodium reabsorption

$T^{T}$ has been a well known fact that the activation of renal efferent nerves exerts a vasoconstrictive effect on renal vessels, especially under stress. However, it remains to be explored whether renal tubular cells have nerve supply and how renal efferent nerves affect tubular function. Many studies on the role of renal nerves in renal function have been performed by means of sectioning or electrical stimulation of the renal or splanchnic nerves. Another method of this study is the recording of the action potential of the renal nerve, in which the renal innervation from brain stem is thought to remain in a more

From the First Department of Internal Medicine, School of Medicine, University of Kanazawa, Kanazawa.

Received for publication July 29, 1968. 
physiological state than in the former methods. Several authors ${ }^{1-5}$ ) have observed the renal nerve discharge under various conditions, mainly in order to study the relationship between renal nerve activity and renal circulation.

In 1964, Gilmore ${ }^{\text {() }}$ observed that the left stellate ganglion stimulation caused acute diuresis and postulated this phenomenon as an effect of withdrawal of renal sympathetic vasoconstrictive nerve discharge. But, one should consider the possibility that the renal sympathetic nerve may have a direct effect on renal tubular function.

In the present study, experiments were carried out to investigate nervous control in the renal tubular function by observing the changes in renal nerve activity, urine volume and urinary constituents before, during and after acute hypertension produced by the electrical stimulation of the left stellate ganglion.

\section{Materials and Methods}

Twenty-five experiments were performed in 7 mongrel dogs weighing 9 to $13 \mathrm{Kg}$. They were anesthetized with a combination of morphine hydrochloride (6 mg./Kg., intramuscularly) and $\alpha$-chloralose (100 mg./Kg., intravenously), with supplementation of $\alpha$-chloralose as required. The trachea was intubated for positive pressure breathing and the required vessels were exposed. Urine was collected by free drainage into graduate cylinders through a polyethylene catheter inserted into the ureter and the femoral arterial blood pressure was recorded with a strain gauge electric manometer (Nihon Kohden MP-4).

Following the collection of preliminary samples of blood and urine, a priming injection of $660 \mathrm{mg}$. of thiosulfate was given intravenously and then sustaining infusion of $50 \mathrm{mg} . / \mathrm{ml}$. of thiosulfate in normal saline was started at a constant rate of $0.5 \mathrm{ml} . / \mathrm{min}$.

Stimulation of the stellate ganglion: After opening the chest by cutting off the posterior angle of the left second rib, the left stellate ganglion was isolated and all rami except the cardiac sympathetic outflow were sectioned. The stellate ganglion was then placed on a shielded bipolar silver electrode connected to an electric stimulator (Nihon Kohden MSE-3). The stimulus was a series of repetitive pulses with a duration of 1 msec., a frequency of $10 \mathrm{cps}$. and an intensity varying 4 to 10 volts.

Recording renal nerve activity: The renal nerve employed was separated from the surrounding tissues in the left renal hilus and was not sectioned. The other nerves remained intact. The action potentials, picked up by a platinum bipolar electrode which was placed around the isolated nerve, were amplified, displayed on a cathode ray oscilloscope (Nihon Kohden VG-7), and also recorded on a multi-channel ink-writing recorder (Nihon Kohden RM-150). The arterial blood pressure was also recorded. In some experiments, an integrator was used for evaluating the changes in action potential. At the end of the experiments, it was ascertained that the employed nerve ran into the renal parenchym.

Chemical procedures: After a 90 to $120 \mathrm{~min}$. equilibrium period following all operative procedures, urine samples were collected every $10 \mathrm{~min}$. and blood 
samples were drawn at the midpoints of urine collection periods from the antecubital vein. When experimental intervention was carried out, the urine for the first $5 \mathrm{~min}$. or the first 2 to $3 \mathrm{ml}$. of urine collected were discarded. The renal clearance of thiosulfate was measured to evaluate glomerular filtration rate. Serum and urine thiosulfate were determined after the method of Brun." ${ }^{7)}$ Serum and urine were analyzed for sodium and potassium concentration using a Coleman flame spectrophotometer, for osmolality by Fiske Model G-62.

\section{Results}

Spontaneous discharges of the renal nerve activity were regularly recorded with the amplitude of 10 to $100 \mathrm{mV}$. They were considered efferent nerve activities since the form of discharge remained unchanged after cutting the nerve distal to the electrode (Fig. 1). The impulses occurred in bursts in synchronization with heart beat, as described in the previous reports on the renal nerve of $\operatorname{dogs}^{5)}$ and cats. ${ }^{1)}$ The impulses were not influenced by respiration in our experimental conditions. However, along with getting into terminal stage, the discharges became frequent to continuous (Fig. 1). The above mentioned recording was excluded from the results of the present study.

The results of stellate ganglion stimulation were as follows: stellate ganglion stimulation resulted in an elevation of mean arterial pressure, a marked widening of pulse pressure and, in most experiments, a decrease in

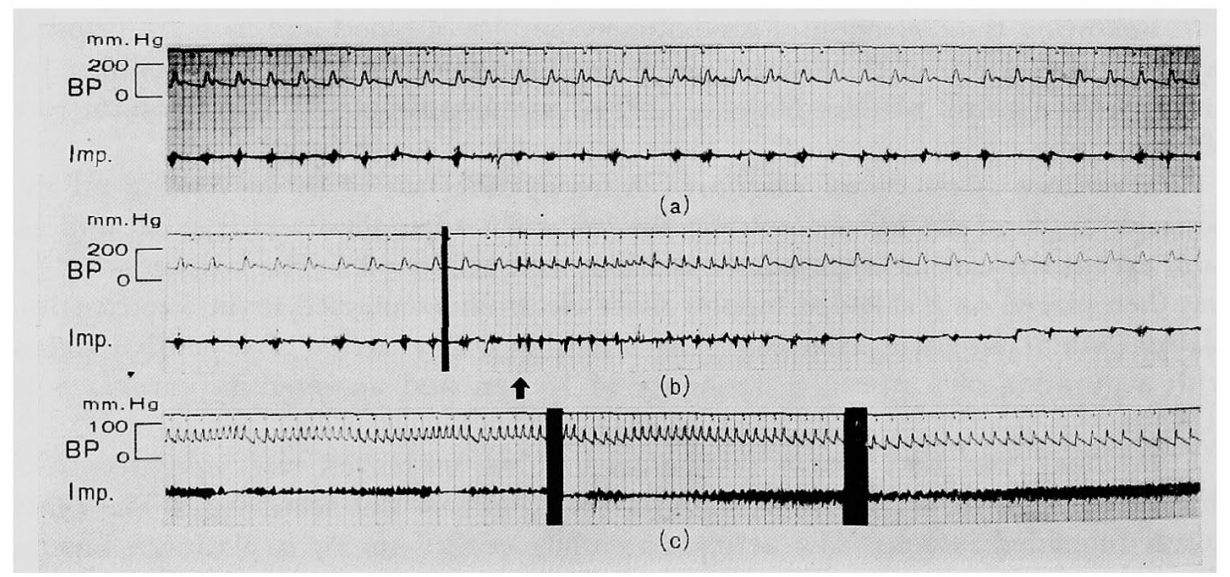

Fig. 1. Renal nerve activity.

(a) Spontaneous discharge. Note that impulses occur in bursts in synchronization with heart beats.

(b) Cutting the nerve distal to the electrode $(\uparrow)$.

(c) Discharges become frequent to continuous, along with getting into terminal stage.

BP: femoral arterial blood pressure. Imp.: impulse of the renal nerve.

(Time: 1 division represents 1 sec.) 


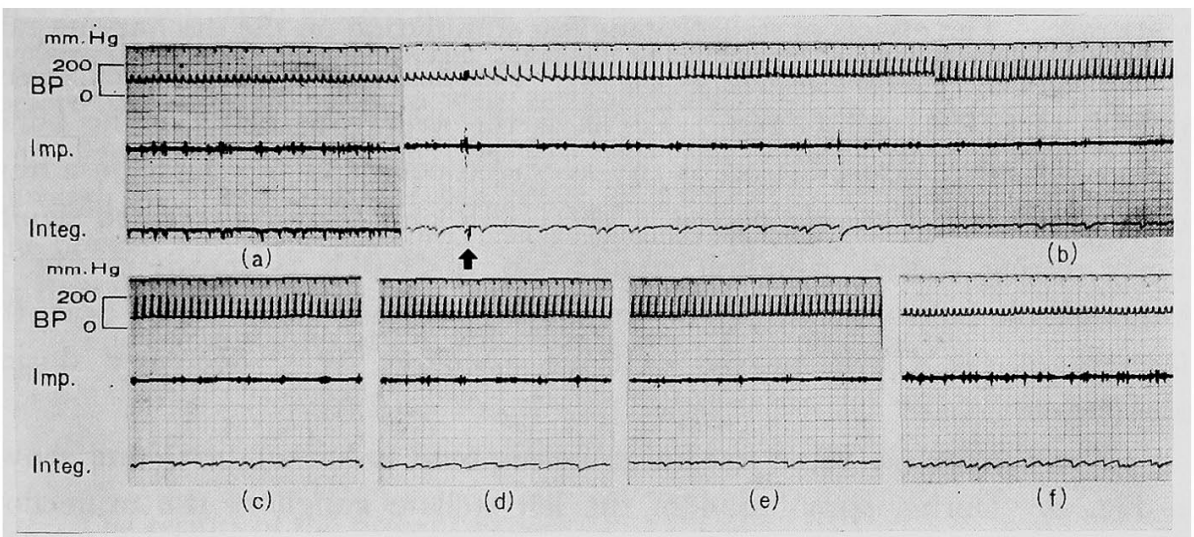

Fig. 2. The change in renal nerve activity following the left stellate ganglion stimulation.

(a) Before stimulation. ( $\uparrow$ ) Beginning of the stimulation

(b)-(e) During stimulation: $3 \mathrm{~min}$. (b), $5 \mathrm{~min}$. (c), $7 \mathrm{~min}$. (d),

9 min. (e), after the beginning of stimulation

(f) At end of stimulation

Integ.: integration of the bursts
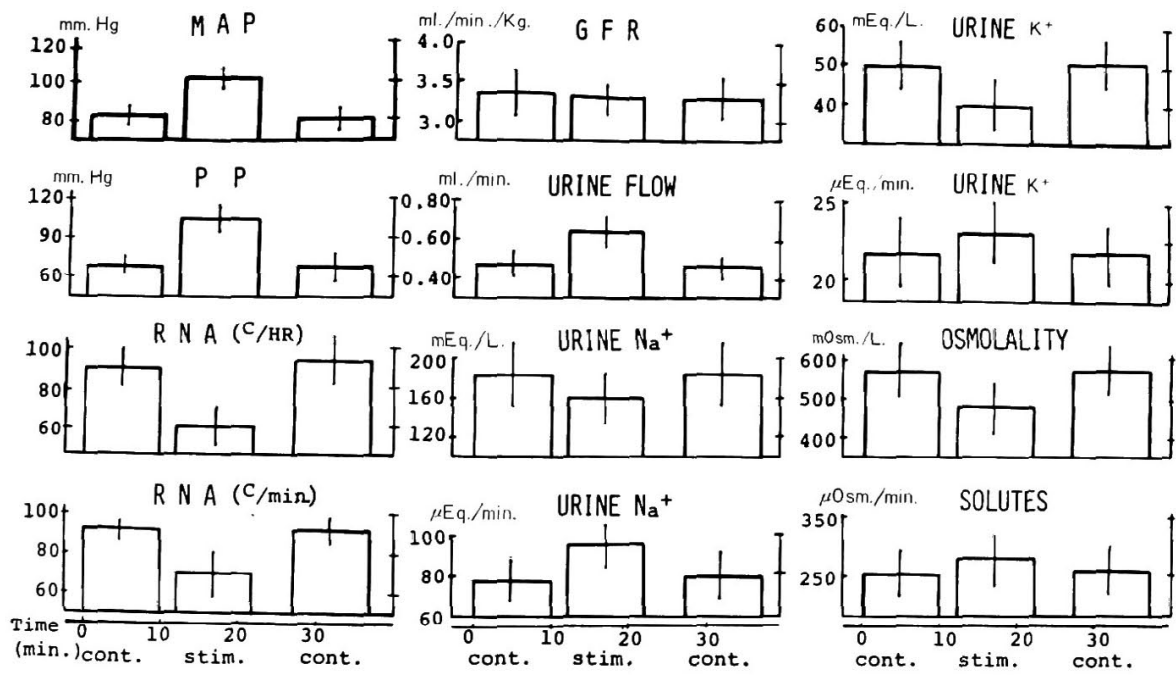

Fig. 3. Experiment illustrating the effect of stellate ganglion stimulation on hemodynamics, renal nerve activity, GFR and urinary constituents.

MAP: mean arterial pressure, PP: pulse pressure, RNA: renal nerve activity, GFR: measured by thiosulfate clearance, Osmolality: total urinary solute concentration, Solutes: total urinary solute excretion. 
heart rate. The effects of stellate ganglion stimulation on the discharging rate of the renal nerve were shown in Fig. 2. The discharging rate was expressed in both of $\mathrm{C} / \mathrm{HR}$ and $\mathrm{C} / \mathrm{min}$; $\mathrm{C} / \mathrm{HR}$ is the averaged counts of the bursts during 100 heart beats, $\mathrm{C} / \mathrm{min}$. is the averaged counts of the bursts in a min. in each $10 \mathrm{~min}$. observing period. The $\mathrm{C} / \mathrm{HR}$ and $\mathrm{C} / \mathrm{min}$. decreascd significantly during stellate ganglion stimulation $(\mathrm{p}<0.01)$, as shown in Fig. 3 . Although the bursts inclined to occur in synchronization with heart beat, the decrease of the $\mathrm{C} / \mathrm{HR}$ proved that the discharge per se decreased during stimulation.

The changes in urine formation in response to stimulation were shown in Fig. 3. During stimulation of the left stellate ganglion, the urine flow $(0.64 \pm 0.08 \mathrm{ml} . / \mathrm{min}$.$) increased in all experiments as compared to the flow$ before and after stimulation $(0.47 \pm 0.06$ and $0.47 \pm 0.05 \mathrm{ml} / \mathrm{min}$., respectively). Sodium and potassium concentration in the serum measured in 2 dogs remained constant in I experimental period for $40 \mathrm{~min}$. In all experiments, except those in $1 \mathrm{dog}$ in which urinary sodium concentration showed a slight increase, urinary concentrations of sodium and potassium declined during stimulation. The changes were statistically significant in 25 experiments $(p<0.01)$. Urinary excretion of sodium and potassium increased significantly $(p<0.01)$ with less change in potassium excretion. The urinary concentration of total solutes decreased $(p<0.01)$ in 25 experiments.

It was noted that the changes were characterized by a rapid onset starting

Table I. Mean Values of Data Measured in This Study

\begin{tabular}{lr|c|c|c} 
& & Control & Stimulation & Control \\
\hline Heart Rate & (per min.) & $100 \pm 12$ & $90 \pm 10$ & $105 \pm 15$ \\
MAP & $(\mathrm{mm} . \mathrm{Hg})$ & $83 \pm 5$ & $102 \pm 6$ & $81 \pm 15$ \\
Pulse Pressure & $(\mathrm{mm} . \mathrm{Hg})$ & $70 \pm 7$ & $115 \pm 11$ & $69 \pm 7$ \\
Urine Volume & $(\mathrm{ml} / \mathrm{min})$. & $0.47 \pm 0.06$ & $0.64 \pm 0.08$ & $0.46 \pm 0.05$ \\
GFR & $(\mathrm{ml} / \mathrm{min} / \mathrm{Kg})$. & $3.36 \pm 0.29$ & $3.33 \pm 0.25$ & $3.30 \pm 0.25$ \\
& $(\mathrm{C} / \mathrm{HR})$ & $92 \pm 5$ & $70 \pm 11$ & $91 \pm 6$ \\
RNA & $(\mathrm{C} / \mathrm{min})$. & $89 \pm 10$ & $60 \pm 10$ & $94 \pm 13$ \\
$\mathrm{U}_{\mathrm{N}^{+}}$ & $(\mathrm{mEq} / \mathrm{L})$. & $182 \pm 33$ & $162 \pm 26$ & $185 \pm 31$ \\
$\mathrm{U}_{\mathrm{K}^{+}}$ & $(\mathrm{mEq} \cdot / \mathrm{L})$. & $50 \pm 6$ & $40 \pm 7$ & $51 \pm 6$ \\
Osmolality & $(\mathrm{mOsm} / \mathrm{L})$. & $560 \pm 63$ & $480 \pm 67$ & $576 \pm 60$ \\
$\mathrm{U}_{\mathrm{Na}^{+}}$ & $(\mu \mathrm{Eq} \cdot / \mathrm{min})$. & $78 \pm 11$ & $96 \pm 12$ & $79 \pm 12$ \\
$\mathrm{U}_{\mathrm{K}^{+}}$ & $(\mu \mathrm{Eq} \cdot / \mathrm{min})$. & $22 \pm 3$ & $23 \pm 2$ & $22 \pm 2$ \\
Solutes & $(\mu \mathrm{Osm} / \mathrm{min})$. & $255 \pm 39$ & $285 \pm 36$ & $260 \pm 39$
\end{tabular}

MAP : mean arterial blood pressure, GFR : thiosulfate clearance,

RNA : renal nerve activity, $\mathrm{C} / \mathrm{HR}$ and $\mathrm{C} / \mathrm{min}$ : : see in the text, $\mathrm{U}$ : urine,

Osmolality: total urinary solute concentration, Solutes: total urinary solute excretion. 
in $\frac{1}{2}$ to $1 \mathrm{~min}$. from the beginning of stimulation and by a rapid disappearance within, at most, $5 \mathrm{~min}$. from the cessation of stimulation. The values measured before and after stimulation were nearly equal.

The estimation of glomerular filtration rate revealed no significant changes through each experimental procedure $(0.2<\mathrm{p}<0.5)$ as shown in Fig. 3. In the present study, the thiosulfate concentration in the serum ranged from 14.5 to $61.9 \mathrm{mg} . / 100 \mathrm{ml}$.

The mean values of the above data are shown in Table I.

\section{Discussion}

The results of the present experiments revealed increases in urine flow and in excretion of sodium, potassium and total solutes ; decreases in urinary concentration of these solutes during stellate ganglion stimulation, without any significant change in glomcrular filtration rate. These results are similar to that of Gilmore's observation. Among humoral or neural factors as basic mechanisms of the diuresis, Gilmore ${ }^{6)}$ claimed the preponderance of neural reflex via baroreceptors to vasoconstrictive renal nerve.

In this report, the discrepancy between the change in glomerular filtration rate and the change in the rate of urine formation could be analysed considering the effects on renal function of the changes in systemic hemodynamics, humoral factor and renal nerve activity produced by stellate ganglion stimulation.

Changes in systemic hemodynamics: It has been generally accepted that the changes in renal arterial pressure and renal arterial pulse pressure within physiological range have little influence on renal blood flow and filtration rate. ${ }^{8)-10)}$ The question raised is whether systemic hemodynamic changes produced by stellate ganglion stimulation are enough to influence urinary excretion directly. There have been many reports concerning the relationship between urinary excretion and renal hemodynamics. For example, Gesell11) believed, from his experiments on dogs, that the reduction in pulse pressure caused diminution in urine flow and chloride excretion. Selkurt ${ }^{12}$ reported that the level of the mean arterial pressure had a dominant role in the electrolytes and water handling; that is, decreases and increases in the excretion of electrolytes and water were paralleled with the direction of change of the mean pressure. He concluded that renal interstitial pressure might influence renal tubular function. Thurau and Deetjen ${ }^{13)}$ reported that an increase in urine flow and a decrease in urinary osmotic pressure followcd an increase in the pressure at which the blood perfused the kidney (Druckdiurese), and attributed the cause of this phenomenon to an increase in medullary blood flow, while total renal blood flow and, presumably, cortical blood flow remained relatively 
constant in this circumstance.

In spite of these evidences, the hemodynamic changes as presented in our experiments do not appear to play a role on the diuresis in response to stellate ganglion stimulation according to the following facts: (a) in Gesell's report, ${ }^{11}$ the time lapse between the change of pulse pressure and the onset of the change in urinary excretion was not constant and was longer than that observed in the present experiments; in Selkurt's experiment, ${ }^{12)}$ the reduction of pulse pressure had no effect on renal function within a wide range of mean arterial pressure ; (b) according to Gottschalk's report, ${ }^{14)}$ the change in renal arterial mean pressure through 40 to $140 \mathrm{~mm} . \mathrm{Hg}$ did not affect renal interstitial pressure ; (c) the experimental condition in the present report can not be compared with that of Druckdiurese ${ }^{13)}$ which was observed in the denervated kidney and $10 \mathrm{~min}$. after an elevation of perfusion pressure ; (d) diuresis induced by stellate ganglion stimulation was characterized by a rapid onset, at the least $\frac{1}{2} \mathrm{~min}$. and a rapid disappearance, within 5 min. after cessation of stimulation; and (e) Gilmore reported that the diuresis induced by stellate ganglion stimulation decreased significantly subsequent to vagotomy, whereas changes in mean arterial pressure before and after vagotomy did not show a significant difference in response to stimulation.

Humoral factors: The next question is whether the stellate ganglion stimulation may be accompanied by changes in the secretion of $\mathrm{ADH}$ and aldosterone which affect renal tubular function. Although the elevation of the left atrial, ${ }^{11)-17)}$ the right atrial and the caval venous pressure ${ }^{18}$ has been conceived to result in a decrease of ADH secretion and an increase of urinary excretion of aldosterone, respectively, it is known that the stellate ganglion stimulation lowers the atrial pressure. ${ }^{6,19)}$ The changes in urine flow and urinary constituents during stellate ganglion stimulation cannot be explained by the alteration of aldosterone secretion. Moreover, these humoral factors do not appear to cause the stellate ganglion stimulation diuresis characterized by rapid respondence, because the changes in urinary excretion of electrolytes due to these two hormones showed a slow onset and a relatively gradual disappearance. ${ }^{15), 18)}$

Renal nerve activity: From the previous discussion, it is strongly suggested that the stellate ganglion stimulation diuresis is associated with changes in renal nerve discharges. The acutely produced diuresis in the present experiments was characterized by its rapid respondence and each measurement shortly after stimulation was close to that before stimulation. In addition, renal nerve discharges decreased significantly during the stimulation. These facts are sufficient to support the hypothesis that a decrease in renal nerve discharge causes increases in urinary excretion of solutes and urine flow, decreases in concentration of urinary solutes which look like water diuresis except in rapid 
respondence. An inference is drawn that an influence on change in renal nerve activity might be through renal tubular function, rather than glomerular filtration rates, since glomerular filtration rate before, during and after stimulation showed no significant changes in the present experiments. If so, how does the renal nerve act on the renal tubular function? Although the mechanism of dilution of the urine has been less adequately described, the following mechanisms might operate in the present experimental procedures: 1) depression in reabsorption of sodium in the proximal tubule, 2) inadequacy of sodium transport in the ascending limb of Henle's loop, 3) change in blood flow through the vasa recta, 4) depression in distal tubular reabsorption of sodium.

We shall first assume that a decrease in renal nerve activity causes a decrease in sodium reabsorption in the proximal tubule. Under this condition, total water and sodium flowing through the tubule distal to Henle's loop are supposed to increase and this results in an increase in sodium excretion and a decline in the sodium concentration in the urine. Since a major fraction of filtered potassium is reabsorbed in the proximal tubule and the most of potassium in the urine is presumably excreted by cation exchange of sodium for potassium in the distal tubule, ${ }^{20), 211}$ potassium excretion may increase under the above assumed condition. The changes of urinary constituents in the present experiments are consistent with this assumption. It has been known that a depression in proximal tubular sodium reabsorption occurs in the natriuresis after a large dose of isotonic saline or a hypertonic saline load, ${ }^{22}$ but it remains to be resolved whether the identical mechanism operates in stellate ganglion stimulation diuresis.

A similar urine may be excreted following either interference of sodium transport in the ascending limb of Henle's loop or a change in blood flow in the vasa recta, through the depression of the medullary osmotic gradient. However, both of these mechanisms could not be supposed to operate when such rapid changes in urinary constituents are observed as in the present experiments.

Depression of the sodium reabsorption in the distal tubule may result in an increase in sodium excretion and a decrease in potassium excretion, if $\mathrm{H}^{+}$ should not be exchanged for $\mathrm{Na}^{+}$than $\mathrm{K}^{+}$in the distal tubule.

By electron-microscopy, Hiraba ${ }^{23)}$ examined renal tubular cells after cutting the renal nerves, and pointed out that the proximal tubular cells began to degenerate in 2 hours after denervation but that the other tubular portions distal to Henle's loop revealed no changes in longer periods. These results are reasonably appreciated from the facts that microscopic observations ${ }^{24), 251}$ showed rich nerve supplies in the proximal tubular region and sparse supplies in the distal portions. 
As discussed above, an assumption that the nervous control operates on renal tubular function especially in proximal tubules, is compatible with the morphological findings, although it is not denied that the changes in urine flow and urinary constituents during acute hypertension might be secondary to the minimal change in glomerular filtration rate undetectable by the present method.

\section{References}

1. Engelhorn, R.: Arch. Exp. Path. Pharmakol. 231: 219, 1957.

2. Dieter, E.: Pfüger Arch. Ges. Physiol. 270: 215, 1960.

3. McGubbin, J. W. and Page, I. H.: Circulat. Res. 6: 816, 1958.

4. Yamakawa, J. and Ishiko, T.: J. Physiol. Soc. Japan 24: 537, 1962 (in Japanese).

5. Takeuchi, J., Ino, S., Hanada, S., Sakai, S., Ohya, N., and Kitamura, K.: Jap. Heart J. 6: $543,1965$.

6. Gilmore, J. P.: Circulat. Res. 14: 301, 1964.

7. Brun, C. J.: J. Lab. Clin. Med. 35: 152, 1950.

8. Selkurt, E. E., Hall P. W., and Sepencer, M. P.: Am. J. Physiol, 159: 369, 1949.

9. Pitts, R. F. and Duggan, J. J.: J. Clin. Invest. 29: 372, 1950.

10. Forster, R. P. and Meas, J. P.: Am. J. Physiol. 150: 534, 1947.

11. Gesell, R. A.: Am. J. Physiol. 32: 70, 1913.

12. Selkurt, E. E.: Circulation 4: $541,1951$.

13. Thurau, K. and Deetjen, P.: Pfüger Arch. 274: 567, 1962.

14. Gottschalk, C. W.: Am. J. Physiol. 163: 716, 1950.

15. Henry, J. P., Gauer, O. H., and Reeves, J. L.: Circulat. Res. 4: 85, 1956.

16. Gauer, O. H., Henry, J. P., and Sieker, H. O.: Prog. Cardiovas. Dis. 4: 1, 1961.

17. Ledsome, J. R., Linden, R. J., and O.Connor, W. J.: J. Physiol. 159: 87, 1961.

18. Davis, J. O., Kliman, B., Yandopoulos, N. A., and Peterson, R. E.: J. Clin. Invest. 37: 1783, 1958.

19. Anzola, J. and Rushmer, R. F.: Circulat. Res. 4: 302, 1956.

20. Watson, J. F.: J. Clin. Invest. 45: 1341, 1966.

21. Davidson, D. G., Levinsky, N. G., and Berliner, R. W.: Am. J. Physiol. 37: 548, 1958.

22. Rector, F. C., Gieson, G. U., Kill, F., and Seldin, D. W.: J. Clin. Invest 43: 341, 1964.

23. Hiraba, K.: Jap. J. Nephrol. 2: 121, 1960 (in Japanese).

24. de Muylder, C. G.: The "Neurility" of the Kidney, Oxford, Blackwell, p. 24, 1952.

25. Mitchell, G. A. G.: Acta anat., Basel 13, 1951. 